\title{
Impact of the COVID-19 Pandemic on Primary Health Care Disease Incidence Rates: 2017 to 2020
}

\author{
Antoni Sisó-Almirall, $M D, P b D^{1,2,3}$ \\ Belcbin Kostov, MSc, $\mathrm{PbD}^{1,2,4}$ \\ Encarna Sánchez, BBA ${ }^{1}$ \\ Jaume Benavent-Àreu, $M D^{1,2}$ \\ Luis González-de Paz, MSc, PbD ${ }^{1,2}$ \\ ${ }^{1}$ Consorci d'Atenció Primària de Salut Barcelona \\ Esquerra (CAPSBE), Barcelona, Spain \\ ${ }^{2}$ Primary Healthcare Transversal Research \\ Group, Institut d'Investigacions Biomèdiques \\ August Pi i Sunyer (IDIBAPS), Barcelona, Spain \\ ${ }^{3}$ Department of Medicine, Faculty of Medicine, \\ Universitat de Barcelona, Barcelona, Spain \\ ${ }^{4}$ Department of Statistics and Operations \\ Research, Universitat Politècnica de Catalunya \\ (UPC), Barcelona, Spain
}

\begin{abstract}
We assessed the impact of the COVID-19 pandemic in Spain on new cases of diseases and conditions commonly seen in primary care. In 2020, there were significant reductions from 2017-2019 in the annual incidences of hypertension (40\% reduction), hypercholesterolemia (36\%), type 2 diabetes (39\%), chronic kidney disease (43\%), ischemic heart disease (48\%), benign prostatic hypertrophy (38\%), osteoporosis (40\%), hypothyroidism (46\%), chronic obstructive pulmonary disease (50\%), alcohol use disorder (46\%), benign colon polyps and tumors (42\%), and melanomas (45\%). Prioritization of COVID-19 care changed the physician-patient relationship to the detriment of face-to-face scheduled visits for chronic disease detection and monitoring, which fell by almost 41\%. To return to prepandemic levels of diagnosis and management of chronic diseases, primary health care services should reorganize and carry out specific actions for groups at higher risk.
\end{abstract}

\section{VISUAL ABSTRACT}

Ann Fam Med 2021;20:63-68. https://doi.org/10.1370/afm.2731.

Annals "Online First" article.

\section{INTRODUCTION}

$\mathrm{T}$ The SARS-CoV-2 coronavirus pandemic has placed unprecedented stress on health systems globally. Spain has experienced $20.3 \%$ excess mortality (4th-highest in Europe) compared with the mean during 2016-2019, ${ }^{1}$ with pandemic fallout leading to radical changes in primary health care. Primary care centers have detected more than $80 \%$ of cases and have redirected their efforts to the prevention, diagnosis, and monitoring of COVID-19 cases and their contacts. ${ }^{2}$ They are also responsible for the early detection of complications and mass testing of vulnerable patients in nursing homes and schools. The prioritization of COVID19 care has changed the physician-patient relationship, reflected by widespread use of teleconsultations, e-mail consultations, and telemonitoring to avoid face-to-face care and minimize the risk of contagion. ${ }^{3}$ COVID-19 preventive measures (especially home confinement, curfews, and physical distancing), the fear of contagion, and the transformation of family medicine services to respond to the pandemic may however have substantially reduced care for chronic noncommunicable diseases and cancer. ${ }^{4,5}$ We aimed to assess the impact of prioritizing care for COVID-19 patients on detection and diagnosis of diseases and conditions commonly seen and managed in primary care, comparing 2020 with 2017-2019.

\section{METHODS}

We undertook a retrospective longitudinal cohort study of variations in the annual incidence of the detection and diagnosis of major cardiovascular risk factors, prevalent chronic diseases and conditions, frequent mental health disorders, and neoplasms during 2017-2020. Data came from a centralized database that records the frequency of the diagnoses studied in 3 primary care centers in Barcelona, Spain. In 2020, the assigned population aged older than 15 years in the 3 centers was 87,478 , with $23.5 \%$ aged 65 years and older. Diagnoses and health problems are recorded by physicians and nurses using the International Classification of Diseases, 10th Revision (ICD-10). We used structured query language (SQL) queries to obtain the aggregated frequency of the conditions studied. As data were anonymized, patient 


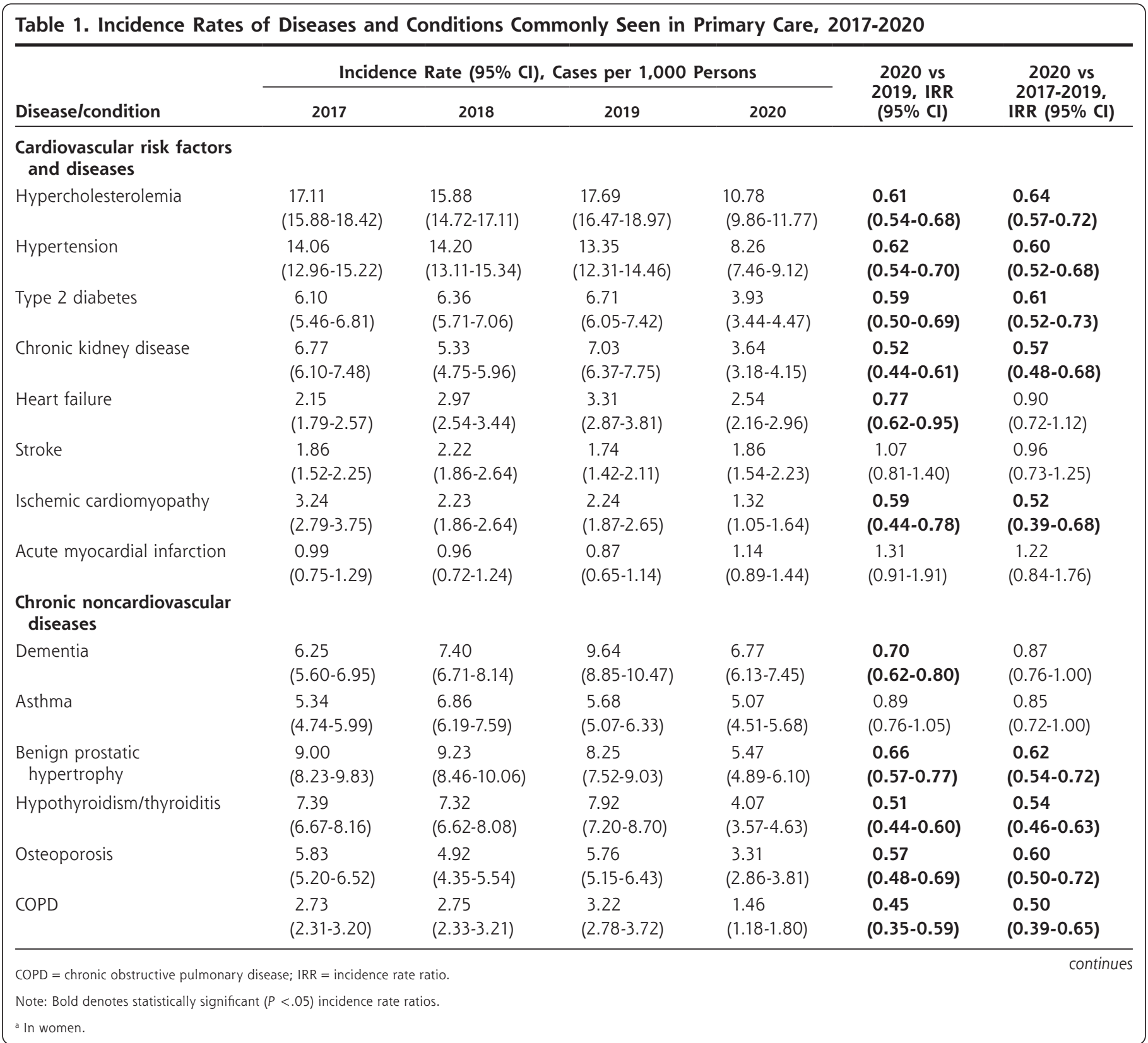

consent was not required, and the study was authorized by the Services Directorate. We calculated the annual incidence rate of cases detected and documented by dividing the number of new cases (having no previous diagnosis in medical records) diagnosed in a specific year by the at-risk population (the number of disease-free people) for each disease category, then multiplying by 1,000 . Incidence rate ratios (IRRs) were used to compare the incidence rate in 2020 with the mean rate in 2017-2019 (primary outcome) and with the rate in 2019 (secondary outcome). We also compared the number of face-to-face scheduled primary care visits in 2020 with that in 2017-2019. Statistical analysis was performed using $\mathrm{R}$ version 3.6.1 (R Project for Statistical Computing) for Windows.

\section{RESULTS}

Numbers of total cases and new cases of cardiovascular diseases, chronic noncardiovascular diseases, mental health disorders, health problems, and cancer during 2017-2020 are shown in Supplemental Table 1.

In 2020, there were significant reductions compared with the 2017-2019 mean in the incidence rates of hypertension $(\mathrm{IRR}=0.60 ; 95 \% \mathrm{CI}, 0.52-0.68)$; hypercholesterolemia (IRR $=0.64 ; 95 \% \mathrm{CI}, 0.57-0.72)$; type 2 diabetes $(\mathrm{IRR}=0.61 ; 95 \% \mathrm{CI}, 0.52-0.73) ;$ chronic kidney disease $(\mathrm{IRR}=0.57 ; 95 \% \mathrm{CI}, 0.48-0.68)$; ischemic cardiomyopathy $(\mathrm{IRR}=0.52 ; 95 \% \mathrm{CI}, 0.39-0.68) ;$ benign prostatic hypertrophy $(\mathrm{IRR}=0.62 ; 95 \% \mathrm{CI}, 0.54-0.72)$; osteoporosis $(I R R=0.60 ; 95 \% \mathrm{CI}, 0.50-0.72)$, hypothyroidism 


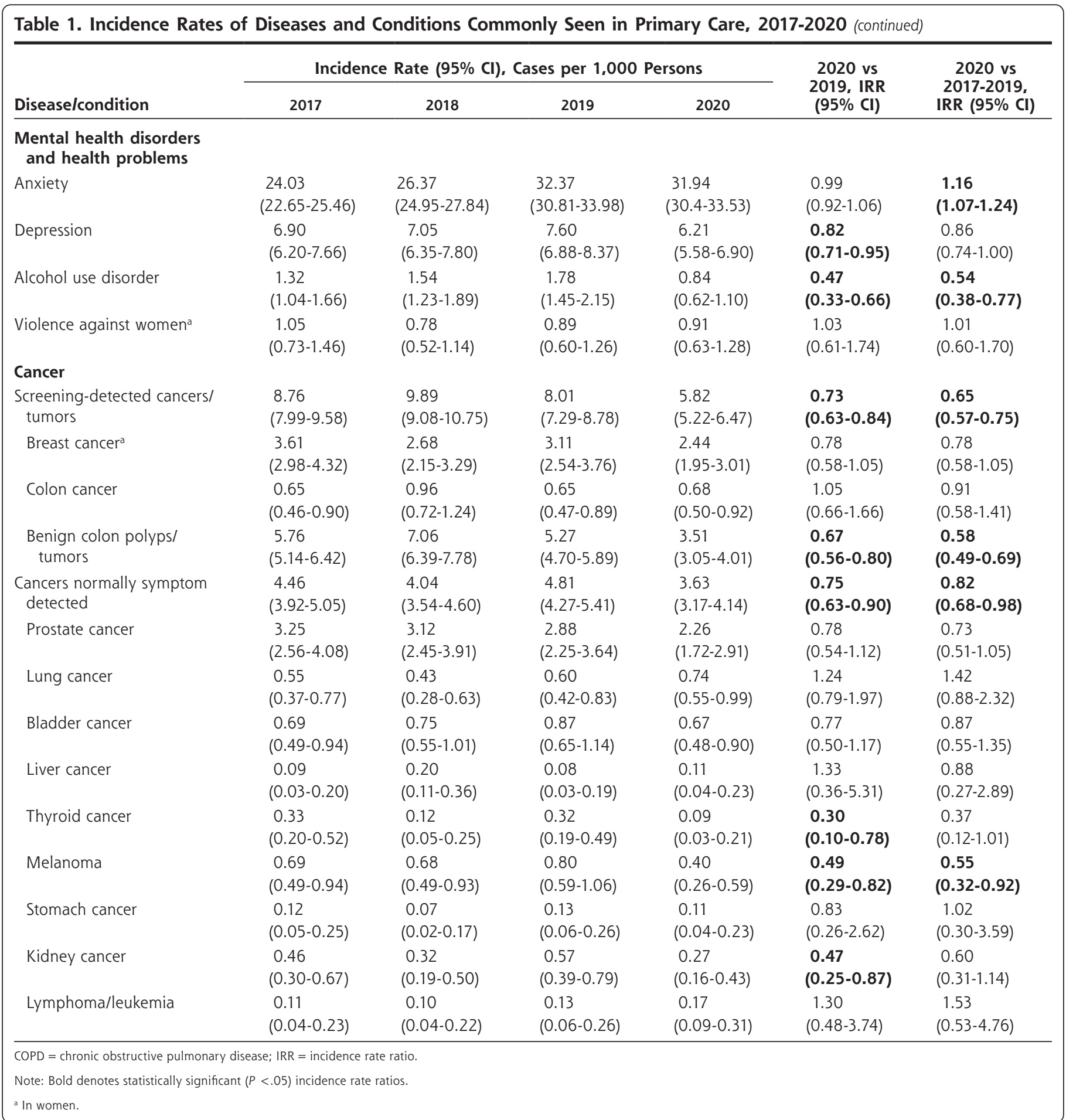

or thyroiditis $(\mathrm{IRR}=0.54 ; 95 \% \mathrm{CI}, 0.46-0.63)$; chronic obstructive pulmonary disease $(\mathrm{IRR}=0.50 ; 95 \% \mathrm{CI}, 0.39$ $0.65)$; alcohol use disorder $(\mathrm{IRR}=0.54 ; 95 \% \mathrm{CI}, 0.38-0.77)$; benign tumors and polyps of the colon (IRR $=0.58 ; 95 \%$ CI, 0.49-0.69); and melanomas (IRR $=0.55 ; 95 \% \mathrm{CI}, 0.32$ 0.92) (Table 1). The reductions ranged from $36 \%$ for hypercholesterolemia to $50 \%$ for chronic obstructive pulmonary disease. The incidence rates of heart failure, dementia, depression, renal cancer, and thyroid cancer were lower in 2020 than in 2019.

The incidences of anxiety disorders (IRR $=1.16 ; 95 \% \mathrm{CI}$, 1.07-1.24) and lung cancer $(\mathrm{IRR}=1.42 ; 95 \% \mathrm{CI}, 0.88-2.32)$ increased during 2020 relative to the mean incidence in 20172019, although the latter increase was not significant. In the 
Figure 1. Trends in incidence rates of selected diseases and conditions commonly seen in primary care, 2017-2020.
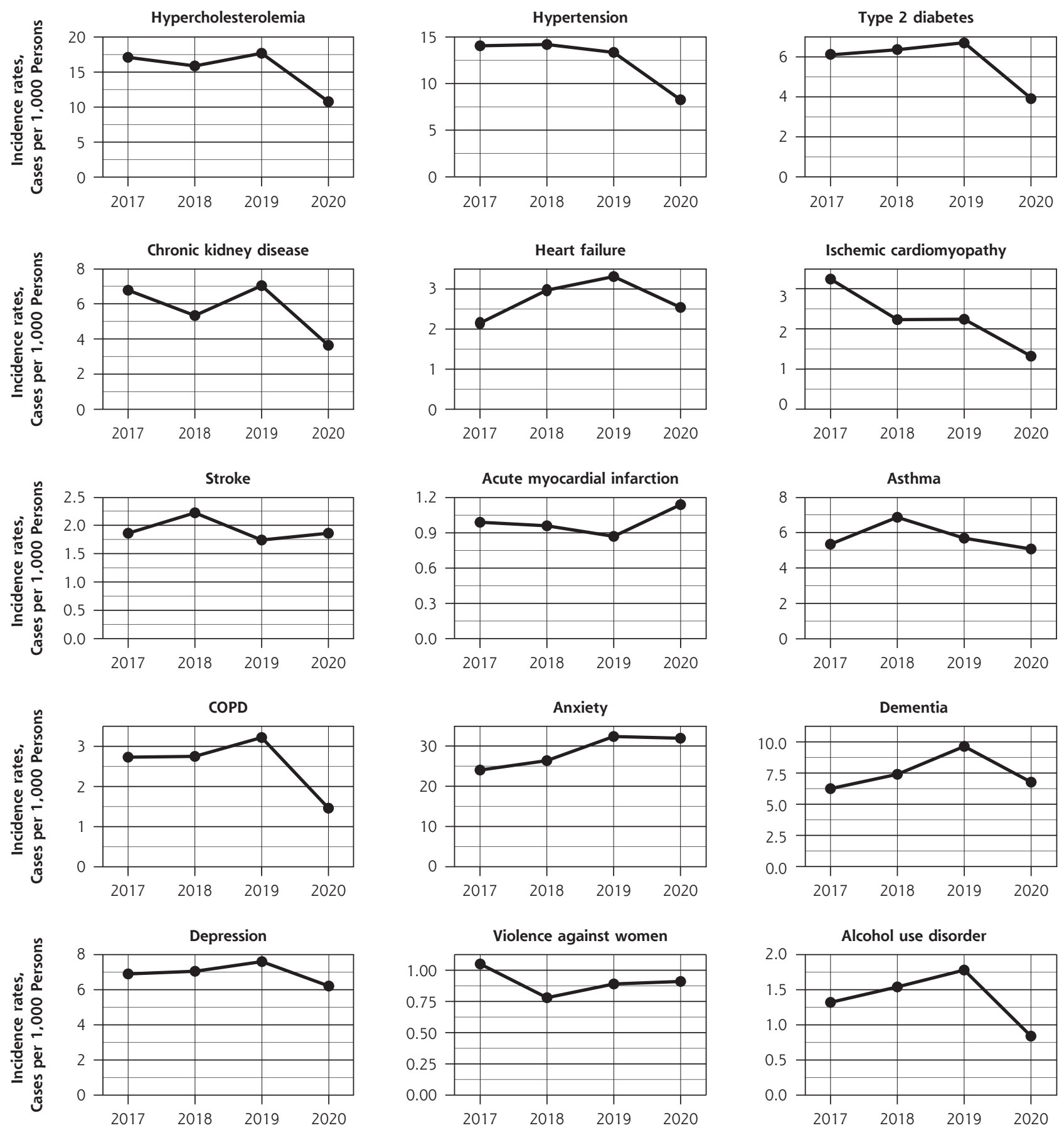

case of anxiety disorders, the higher rate in 2020 was mainly due to persistence of a substantial increase in 2019 compared with 2018. Figure 1 shows the trend in the annual incidence for each of the diseases and conditions studied over the 4 years analyzed.

The number of face-to-face scheduled primary care visits in 2020 (207,102 visits) was $40.9 \%$ lower than the mean in 2017-2019 (350,432 visits).

\section{DISCUSSION}

Our results show a substantial reduction in diagnoses of cardiovascular risk factors, chronic diseases and conditions, and some tumors in 2020 compared with 2017-2019, which may be attributed to organizational changes in primary care services aimed at prioritizing COVID-19 care. Other studies analyzing indicators of chronic disease follow-up during the first 4 months of the pandemic reached similar conclusions. ${ }^{6,7}$ 
Figure 1. Trends in incidence rates of selected diseases and conditions commonly seen in primary care, 2017-2020. (continued)
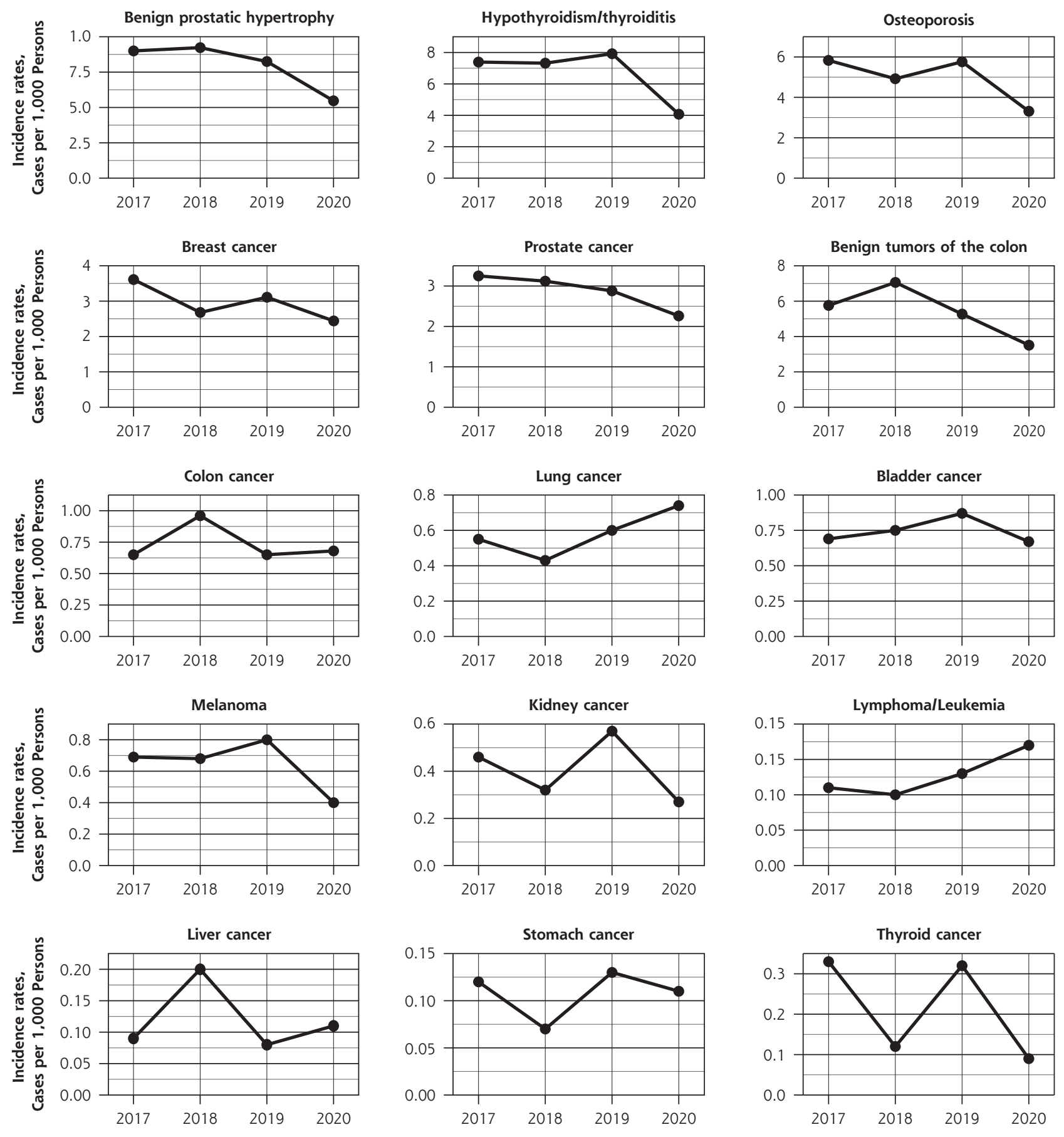

The increased incidence of lung cancer may be linked to the increase in pulmonary radiologic tests (chest radiographs and chest computed tomography) due to the pandemic.

Between February and December 2020, primary care centers in Barcelona were "bunkerized," whereby use of these centers was limited. The new model of care decreasing access and proximity, the loss of scheduled preventive and health promotion visits, and the change from face-to-face visits to virtual consultations all contributed to the reduction in incidence rates. ${ }^{8}$ Minimizing face-to-face contact to protect against COVID-19 may have therefore had a paradoxical detrimental effect on the control of chronic disease. This effect might increase further with the start of the COVID-19 vaccination campaign in January 2021, which is being managed 
by primary care professionals. The plateauing of the rise in anxiety disorders is also consistent with the current situation and the underreporting of most types of diagnoses. ${ }^{9}$

Returning to the level of detection and control of chronic diseases before the pandemic will require a substantial increase in primary care physicians and nurses, a return to face-to-face visits, a reorganization of telecare, and the promotion of proactive care for patients with the greatest comorbidity in European Union countries.

Read or post commentaries in response to this article.

Key words: COVID-19; SARS-Cov-2; pandemics; primary care; chronic conditions; underdiagnosis; telemedicine; delivery of health care; health services accessibility; organizational change; population health

Submitted February 9, 2021; submitted, revised, June 4, 2021; accepted June 21, 2021.

\section{Supplemental materials}

\section{References}

1. Eurostat. Excess mortality - statistics. Updated Jul 14, 2021. Accessed May 15, 2021. https://ec.europa.eu/eurostat/statistics-explained/index.php?title= Excess_mortality_-_statistics\#Further_releases
2. Catalan Agency for Health Quality and Evaluation (AQuAS). Updated SARSCoV-2 data. Accessed Jan 25, 2021. https://aquas.gencat.cat/ca/actualitat/ ultimes-dades-coronavirus/index.html

3. Greenhalgh T, Koh GCH, Car J. Covid-19: a remote assessment in primary care. BMJ. 2020;368:m1182.

4. Uyl-de Groot CA, Schuurman MS, Huijgens PC, Praagman J. Fewer cancer diagnoses during the COVID-19 epidemic according to diagnosis, age and region [published online ahead of print December 11, 2020]. TSG. 2020:1-8. 10.1007/s12508-020-00289-1

5. Dinmohamed AG, Visser O, Verhoeven RHA, et al. Fewer cancer diagnoses during the COVID-19 epidemic in the Netherlands. Lancet Oncol. 2020;21(6): 750-751.

6. Coma E, Mora N, Méndez L, et al. Primary care in the time of COVID-19: monitoring the effect of the pandemic and the lockdown measures on 34 quality of care indicators calculated for 288 primary care practices covering about 6 million people in Catalonia. BMC Fam Pract. 2020;21(1):208.

7. Zheng NS, Warner JL, Osterman TJ, et al. A retrospective approach to evaluating potential adverse outcomes associated with delay of procedures for cardiovascular and cancer-related diagnoses in the context of COVID-19. J Biomed Inform. 2021;113:103657.

8. Schers H, van Weel C, van Boven K, Akkermans R, Bischoff E, olde Hartman T. The COVID-19 pandemic in Nijmegen, the Netherlands: changes in presented health problems and demand for primary care. Ann Fam Med. 2021; 19(1):44-47.

9. Fancourt D, Steptoe A, Bu F. Trajectories of anxiety and depressive symptoms during enforced isolation due to COVID-19 in England: a longitudinal observational study. Lancet Psychiatry. 2021;8(2):141-149. 